\title{
C Proposed Physician Payment Schedule for 2013: Guarded Prognosis for Interventional Pain Management
}

Laxmaiah Manchikanti, MD', Vijay Singh, MD², David L. Caraway, MD,

Ramsin M. Benyamin, MD ${ }^{4}$, Frank J.E. Falco, MD ${ }^{5}$, and Joshua A. Hirsch, MD

From: 'Pain Management Center of Paducah, Paducah, KY; and University of Louisville, Louisville, KY; ${ }^{2}$ Spine Pain Diagnostics Associates, Niagara, Wl;3St. Mary's Pain Relief Center, Huntington, WV; ${ }^{4}$ Millennium Pain Center, Bloomington, IL, and University of Illinois, Urbana-Champaign, IL; and ${ }^{5}$ Mid Atlantic Spine \& Pain Physicians, of Newark, Newark, $\mathrm{DE}$, and Temple University Hospital, Philadelphia, PA ${ }^{6}$ Massachusetts General Hospital, and Harvard Medical School, Boston, MA.

Author Affiliation information on pp. E624-E625

Address correspondence: Dr. Laxmaiah Manchikanti Pain Management Center of

Paducah

2831 Lone Oak Raod

Paducah, KY 42003

E-mail:drlm@thepainmd.com

Conflict of interest: None.

Manuscript received: 09/02/2012

Accepted for publication $09 / 12 / 2012$
As happens every year, on July 1, 2012, the Centers for Medicare and Medicaid Services issued a proposed policy and payment rate for services furnished under the Medicare physician fee schedule for 2013. The proposed rule would provide certified registered nurse anesthetists to practice independent interventional pain management. Other issues, though no less important, include a $27 \%$ sustainable growth rate formula cut in reimbursement, along with a $2 \%$ sequester, which could lead to a potential cut of $29 \%$.

Since the inception of Medicare programs in 1965, several methods have been used to determine the amounts paid to physicians for each covered service. The sustainable growth rate was enacted in 1997 to determine physician payment updates under Medicare Part B. Its intent was to reduce Medicare physician payment updates to offset the growth and utilization of physician services that exceed gross domestic product growth. This is achieved by setting an overall target amount of spending for physicians' services and adjusting payment rates annually to reflect differences between actual spending and the spending target. Since 2002, the sustainable growth rate has annually been used to recommend reductions in Medicare reimbursements. Payments were cut in 2002 by $4.8 \%$. Since then, Congress has intervened on multiple occasions to prevent additional cuts from being imposed.

In this manuscript, we will describe important proposed changes to the physician fee schedule. Additionally, the impact of multiple changes on interventional pain management will be briefly described.

Key words: Health policy, physician payment policy, physician fee schedule, Medicare, sustained growth rate formula, interventional pain management, regulatory reform.

Pain Physician 2012; 15:-E615-E627 $\mathrm{n}$ the routine climate of regulations and bad news for physicians, on July 1, 2012, the Centers for Medicare and Medicaid Services (CMS) issued a proposed policy and payment rate for services furnished under the Medicare physician fee schedule for 2013 (1-7). The proposed rule also advocates changes to several quality reporting initiatives, as well as electronic prescribing, multiple procedure payment reductions, care coordination, and various other changes. However, this year's proposed rule is of significant importance for interventional pain physicians. A bombshell provision in this proposed rule is to allow certified registered nurse anesthetists (CRNAs) to practice independent interventional pain management $(1,8)$. Other issues, though no less important, include a $27 \%$ sustainable growth rate (SGR) formula cut in reimbursement, topped by a $2 \%$ sequester $(9,10)$. While SGR has been a looming issue for many years now, and physicians are used to disappointments each year, the independent practice of nurse anesthetists to perform interventional techniques, and sequester are new phenomena. 
Much like the SGR, the budget sequester that also threatens the program in 2013 is cryptically named and arbitrary in nature (10). The sequester is a governmentwide spending reduction plan for the next decade. It is not based on health policy, but is a budgeting mechanism functioning as a meat axe or buzz saw, eviscerating federal agency budgets over a period of 9 years, unlike the precision and elegance of a surgical scalpel (10). However, Social Security and Medicaid are exempt from sequester; all other areas of the government will share the budget cuts. In addition, enrollees in Medicare are considered to be safe since it does not cut their benefits, but physicians and other health professionals providing their health care will suffer, which ultimately may also result in suffering for Medicare enrollees.

In 2013, sequestration will begin to hit those contracted to provide Medicare services, including physicians, hospitals, nurses, therapists, medical suppliers, and drug providers. Payments will be reduced $2 \%$, resulting in savings of $\$ 11$ billion in 2013 and $\$ 123$ billion through 2021. Otherwise, proposed payment rates, either in a facility or nonfacility setting, show modest cuts, ranging as high as $8 \%$ for nonfacility settings to $5 \%$ for facility settings.

The additional key provisions of the proposed rule for calendar year 2013 are summarized below.

- Physician Quality Reporting System (PQRS): For 2013 and 2014, CMS proposes to include 264 individual measures for PQRS, along with 26 measure groups for 2013, 4 more than 2012. For the purpose of aligning various CMS quality programs, CMS proposes to align PQRS measures available for EHR (electronic health records)-based reporting with measures under the EHR Incentive Program.

Successful participation in the 2013 or 2014 PQRS program will result in a $0.5 \%$ incentive payment, based on estimated total allowed charges for all covered services during the reporting period. Qualifying for an incentive payment in 2013 and 2014 allows an eligible professional (EP) to also avoid penalties in 2015 and 2016.

However, there are no quality measures available for interventional pain management.

- Medicare Shared Savings Program (MSSP): To promote alignment of the MSSP and PQRS programs, CMS proposes to use the same quality measures in both programs as well as the reporting criteria that are used in the PQRS incentive and payment adjustment program. MSSP EPs are also on the hook for PQRS penalties, and therefore must comply with the MSSP requirements for satisfactory participation in the PQRS Group Practice Reporting Option (GPRO) Web interface.

- Value-Based Payment Modifier (VBM): Created by ObamaCare, the VBM will lead to payment adjustments based on a comparison of physicians' costs and quality. The proposal is budget-neutral, so increases in Medicare payment rates for some physicians will be offset by reductions for others. The VBM must be applied to some physicians in 2015 and to all physicians by 2017.

- In the first year, CMS is proposing to apply the adjustment only to physicians who practice in groups of 25 or more. Physicians in the affected groups could avoid any adjustment in their payments if the group signed up for and successfully participated in one of several PQRS options.

- $\quad$ For groups that were not successful PQRS participants, 2015 Medicare payment rates would be cut by $1 \%$. Those that were successful could either take a zero payment adjustment in 2015 or opt to be judged through a 3-tiered system that would incorporate both costs and quality. Low tier participants would face $1 \%$ payment cuts; those in the middle would see no change; and those in the high tier would receive an asyet-undetermined increase.

- Physician Feedback Reporting Program: For a preview of the impact of the VBM on physicians' payments, beginning in the fall of 2014 physicians in groups of 25 or more will receive a confidential feedback report that includes the VBM that will be applied to the physician's payments in 2015. Officially known as Quality and Resource Use Reports (QRUR), these reports will compare quality and resource use among physicians. Current versions of the report rely on administrative claims data and PQRS measures (where available) to measure quality. Costs are tied to per-patient costs for all services and to 4 chronic conditions to be used in the VBM program. In concert with state and specialty medical society staff, the AMA has been engaged in an extensive review of the QRURs and has offered CMS many suggestions for making the reports more fair and useful. 
- Physician Compare: CMS continues to phase in expansion of the Physician Compare Web site by proposing to make public the quality measure performance rates for group practices participating in the 2013 PQRS Web interface GPRO or the MSSP. The 2013 PQRS GPRO Web interface option is only available to practices comprising 25 or more EPs, with different reporting criteria for groups of 25-99 EPs and groups of 100 or more EPs. Patient experience survey data based on 2013, such as the Clinician and Group Consumer Assessment of Healthcare Providers and Systems (CG-CAHPS), will be posted no earlier than 2014 for group practices participating in any PQRS GPRO (not just Web interface reporting). CMS will administer and collect patient experience survey data on a sample of group practices' beneficiaries in 2013. The agency will also publicly report on Physician Compare, beginning in 2013, and patient experience data for Accountable Care Organizations (ACOs) participating in the MSSP. CMS proposes to lower the minimum threshold for reporting performance information on Physician Compare from 25 to 20 patients, and seeks input on proposals to add additional group-level and individual quality measures for public reporting.

- Electronic Prescribing (eRx) Incentive Program: CMS proposes improvements to the eRx program as follows:

- Addition of 2 hardship exemption categories tied to participation in the meaningful use EHR incentive program making it easier to avoid eprescribing penalties in 2013 and 2014

- Establishment of a process so that physicians encountering problems associated with 2013 e-prescribing incentives and the 2014 penalty program can request CMS' review of their case

- Lower the reporting requirement for eligible group practices comprising 2-24 health care professionals.

In addition, CMS proposes updating certain e-prescribing technological standards under Medicare Part D to improve e-prescribing functionality.

- Care Coordination: CMS outlines the initiatives developed to date to incentivize and promote improved care coordination, including:

- MSSP (Pioneer ACO Model and Advance Payment ACO model)

- Primary Care Incentive Payment (PCIP) Program
- Multi-payer Advanced Primary Care Practice (MAPCP) Demonstration

- Federally Qualified Health Center (FQHC) Advanced Primary Care Practice Demonstration

- Comprehensive Primary Care (CPC) Initiative.

- Practice Expense Equipment Interest Rate: The practice expense (PE) relative value unit (RVU) methodology requires a calculation of equipment cost per minute, including calculation of the interest rate. The interest rate CMS previously used was outdated and applied the same interest rate across all equipment. Consistent with the American Medical Association's recommendation, CMS is proposing a "sliding scale," which is a variable interest rate based on useful life, equipment cost and the Small Business Administration's (SBA) maximum interest rates for different categories of loan size and maturity. This method for interest rates will account for changes in the prime rate or the SBA's formula for maximum allowed interest rates.

- Geographic Practice Cost Indices (GPCls): As required by law, CMS updates the GPCls every 3 years. The next GPCl update will be in 2014, so CMS has not proposed GPCI changes in the current proposed rule, although it notes that the statutory work GPCl floor of 1.00 is set to expire at the end of 2012. As it considers potential future GPCI policy proposals, CMS is analyzing recommendations from a panel convened by the Institute of Medicine (IOM). Several IOM Phase I recommendations are discussed in the current proposed rule. For example, the rule notes that adoption of the IOM recommendation for employee wage index payment locality changes could potentially increase the number of localities from the current 89 to over 3,000. CMS plans to address the IOM Phase I and II recommendations more fully in future rulemaking.

- Multiple Procedure Payment Reduction: CMS would apply a $25 \%$ multiple procedure payment reduction to the technical component of diagnostic cardiovascular and ophthalmology services when these services are furnished by the same physician (or physicians in the same group practice) to the same patient on the same day.

- Elimination of Requirement to Terminate Prepayment Medical Review: The current one-year limit on nonrandom prepayment medical review would be removed. 
- Face-to-Face Requirement for Durable Medical Equipment: The Patient Protection and Affordable Care Act requires that a physician have a faceto-face encounter with a beneficiary during the 6-month period prior to a written order for certain Medicare-covered durable medical equipment. CMS proposes to change this 6-month requirement to no more than 90 days before the order is written or within 30 days after the order is written.

Congressional research surveys in December of 2011 (11) evaluated the Medicare physician payment updates and the SGR system. As is well known, in the first few years of the SGR system, the actual expenditures did not exceed the targets and the updates to the physician fee schedule were close to the Medicare Economic Index (MEI), a price index of inputs required to produce physician services fees. For the next 2 years, in 2000 and 2001, the actual physician fee schedule update was more than twice the MEI for those years. Beginning in 2002, the actual expenditure exceeded allowed targets, and the discrepancy has grown with each year. However, with the exception of 2002, when a $4.8 \%$ decrease was applied, Congress has enacted a series of laws to override the reductions.

There has been a growing consensus among observers that the SGR system is fundamentally flawed and is creating instability in the Medicare program for providers and beneficiaries (11). The SGR system treats all services and physicians equally in the calculation of the annual payment update, which is applied uniformly with no distinction across specialties. In addition, there has been an increased concern that continued declines in physician payment rates, especially among primary care specialists, may potentially jeopardize access to services. However, in contrast to the beliefs of Congress, all specialties are affected. Legislative overrides since 2002 have only provided temporary reprieves from projected reductions in payments under the SGR calculation, requiring even steeper reductions in payment rates in the future.

Table 1 illustrates proposed physician payment rates, however, without cuts at the present time. Consequently, if Congress and the Administration fail to act, the SGR cuts of $27 \%$, sequester of $2 \%$, and any additional cuts CMS makes opposing their final rule, may be devastating to all physicians, specifically interventional pain physicians.

These cuts could lead to serious access to care issues for patients. Medicare physician payments have been nearly frozen for a decade, whereas the cost of caring for patients has increased at least by more than $20 \%$; such cuts will be devastating. Regulations for medicine, specifically for interventional pain management, have been devastating with the proposed implementation of ICD-10, electronic medical records (EMRs), and single-dose vial regulations (12-25). In addition, there are other issues, such as ObamaCare in general (26-30), Patient-Centered Outcomes Research Institute (PCORI) (31-33), comparative effectiveness research $(34,35)$ and Independent Payment Advisory Board (IPAB) (36-38).

Table 2 shows the proposed rule's estimated impact on total allowed charges by specialty. As shown, there is a $3 \%$ reduction for anesthesiology, interventions, and radiology, whereas the reductions are $1 \%$ for interventional pain management with a $1 \%$ increase for physical medicine and rehabilitation, along with physical therapists. Of interest is that increases are much higher for other practitioners, with a 5\% increase for nurse practitioners and a 3\% increase for physician assistants; however, there is a $3 \%$ decrease for clinical psychologists and $4 \%$ decrease for nurse anesthetists and anesthesia assistants.

Unless Congress enacts legislation to override projected SGR changes, physician fees would be reduced by $29 \%$ in calendar year 2013 , including the $2 \%$ sequester. For 2012, a one year freeze to physician payments was estimated to cost $\$ 11$ billion and $\$ 21$ billion over 10 years from 2012 to 2021, according to the Congressional Budget Office (CBO). However, a long-term fix such as repealing the SGR, combined with a freeze in physician pay rates over the next 10 years, would cost approximately $\$ 290$ billion.

The Medicare Payment Advisory Commission (MedPAC), in its recommendations for addressing the SGR and Medicare physician payments to Congress, on October 14, 2011, submitted a recommendation that Congress repeal the SGR system and replace it with a 10 -year schedule of specified updates for the physician fee schedule (39); however, this recommendation drew significant criticism from various specialties. In this recommendation, specifically, primary care practitioners would have a $0 \%$ update over the next 10 years, while nonprimary care practitioners would experience a $5.9 \%$ decline in payment rates the first 3 years and $0 \%$ thereafter. MedPAC estimated this would cost about $\$ 200$ billion over 10 years. Further, it also recommended multiple options to offset this cost, which would spread the impact of reductions across other providers and Medicare beneficiaries. Congress passed a law in February 2012 to avoid the cuts through December 30, 2012 (40). 
Proposed Physician Payment Schedule for 2013

Table 1. 2013 proposed physician payment rates.

\begin{tabular}{|c|c|c|c|c|c|c|c|c|c|}
\hline \multirow[b]{2}{*}{ CPT } & \multirow[b]{2}{*}{ Description } & \multicolumn{2}{|c|}{$2011(C F=\$ 33.9764)$} & \multicolumn{2}{|c|}{$2012(C F=\$ 34.0376)$} & \multicolumn{2}{|c|}{$\begin{array}{l}2013 \text { Proposed without } \\
\text { cut }(C F=\$ 34.0376)\end{array}$} & \multicolumn{2}{|c|}{ \% change from 2012} \\
\hline & & $\begin{array}{l}\text { Non-Facility } \\
\text { (Office) }\end{array}$ & $\begin{array}{c}\text { Facility } \\
\text { (ASC/Hospital) }\end{array}$ & $\begin{array}{l}\text { Non-Facility } \\
\text { (Office) }\end{array}$ & $\begin{array}{l}\text { Facility } \\
\text { (ASCl } \\
\text { Hospital) }\end{array}$ & $\begin{array}{l}\text { Non-Facility } \\
\text { (Office) }\end{array}$ & $\begin{array}{c}\text { Facility } \\
\text { (ASC/Hospital) }\end{array}$ & $\begin{array}{l}\text { Non-Facility } \\
\text { (Office) }\end{array}$ & $\begin{array}{l}\text { Facility } \\
\text { (ASC/ } \\
\text { Hospital) }\end{array}$ \\
\hline 20526 & $\begin{array}{l}\text { Injection, therapeutic } \\
\text { (eg, local anesthetic; } \\
\text { corticosteroid), carpal tunnel }\end{array}$ & $\$ 73.73$ & $\$ 56.74$ & $\$ 74.88$ & $\$ 56.50$ & $\$ 75.22$ & $\$ 56.16$ & $0.5 \%$ & $-0.6 \%$ \\
\hline 20550 & $\begin{array}{l}\text { tendon sheath, ligament } \\
\text { injection }\end{array}$ & $\$ 56.06$ & $\$ 40.77$ & $\$ 57.18$ & $\$ 40.85$ & $\$ 57.52$ & $\$ 40.85$ & $0.6 \%$ & $0.0 \%$ \\
\hline 20551 & Tendon origin/insertion & $\$ 57.08$ & $\$ 42.13$ & $\$ 58.54$ & $\$ 42.21$ & $\$ 59.57$ & $\$ 42.21$ & $1.7 \%$ & $0.0 \%$ \\
\hline 20552 & $\begin{array}{l}\text { Single or multiple trigger } \\
\text { point(s), one or two muscle } \\
\text { group(s) }\end{array}$ & $\$ 52.32$ & $\$ 36.69$ & $\$ 53.78$ & $\$ 37.10$ & $\$ 54.80$ & $\$ 37.78$ & $1.9 \%$ & $1.8 \%$ \\
\hline 20553 & $\begin{array}{l}\text { Single or multiple trigger } \\
\text { point(s), three or more } \\
\text { muscle groups }\end{array}$ & $\$ 59.12$ & $\$ 41.11$ & $\$ 61.61$ & $\$ 41.87$ & $\$ 62.97$ & $\$ 42.55$ & $2.2 \%$ & $1.6 \%$ \\
\hline 20600 & Small joint injection & $\$ 53.00$ & $\$ 38.73$ & $\$ 54.12$ & $\$ 38.46$ & $\$ 54.80$ & $\$ 38.46$ & $1.3 \%$ & $0.0 \%$ \\
\hline 20605 & Intermediate joint injection & $\$ 57.76$ & $\$ 40.77$ & $\$ 57.18$ & $\$ 40.50$ & $\$ 57.18$ & $\$ 40.50$ & $0.0 \%$ & $0.0 \%$ \\
\hline 20610 & Major joint injection & $\$ 76.79$ & $\$ 49.61$ & $\$ 69.78$ & $\$ 49.69$ & $\$ 68.08$ & $\$ 49.69$ & $-2.4 \%$ & $0.0 \%$ \\
\hline 22520 & Vertebroplasty (Thoracic) & $\$ 2,229.53$ & $\$ 523.58$ & $\$ 2,251.93$ & $\$ 512.61$ & $\$ 2,254.65$ & $\$ 503.08$ & $0.1 \%$ & $-1.9 \%$ \\
\hline 22521 & Vertebroplasty (Lumbar) & $\$ 2,187.40$ & $\$ 495.04$ & $\$ 2,241.72$ & $\$ 487.76$ & $\$ 2,257.37$ & $\$ 480.27$ & $0.7 \%$ & $-1.5 \%$ \\
\hline 22522 & Vertebroplasty - Additional & NA & $\$ 231.04$ & NA & $\$ 226.69$ & NA & $\$ 222.61$ & NA & $1.8 \%$ \\
\hline 22526 & IDET, single level & $\$ 2,090.23$ & $\$ 324.81$ & $\$ 2,261.12$ & $\$ 331.53$ & $\$ 2,396.25$ & $\$ 339.35$ & $6.0 \%$ & $2.4 \%$ \\
\hline 22527 & IDET, 1 or more levels & $\$ 1,681.15$ & $\$ 145.76$ & $\$ 1,846.54$ & $\$ 148.74$ & $\$ 1,995.62$ & $\$ 152.49$ & $8.1 \%$ & $2.5 \%$ \\
\hline 27093 & $\begin{array}{l}\text { Injection procedure for HIP } \\
\text { arthrography - without } \\
\text { anesthesia }\end{array}$ & $\$ 189.25$ & $\$ 70.33$ & $\$ 195.04$ & $\$ 70.12$ & $\$ 189.25$ & $\$ 69.78$ & $-3.0 \%$ & $-0.5 \%$ \\
\hline 27095 & $\begin{array}{l}\text { Injection procedure for } \\
\text { HIP arthrography - with } \\
\text { anesthesia }\end{array}$ & $\$ 231.72$ & $\$ 81.88$ & $\$ 241.33$ & $\$ 81.69$ & $\$ 236.56$ & $\$ 81.69$ & $-2.0 \%$ & $0.0 \%$ \\
\hline 27096 & $\begin{array}{l}\text { (G0260) Injection } \\
\text { procedure for Sacroiliac } \\
\text { joint, arthrography }\end{array}$ & $\$ 184.49$ & $\$ 70.67$ & $\$ 171.89$ & $\$ 82.03$ & $\$ 166.44$ & $\$ 85.43$ & $-3.2 \%$ & $4.1 \%$ \\
\hline 62263 & \begin{tabular}{|l|} 
Percutaneous epidural \\
adhesiolysis -2 or 3 days
\end{tabular} & $\$ 708.75$ & $\$ 399.56$ & $\$ 683.48$ & $\$ 340.72$ & $\$ 702.20$ & $\$ 352.63$ & $2.7 \%$ & $3.5 \%$ \\
\hline 62264 & $\begin{array}{l}\text { Percutaneous epidural } \\
\text { adhesiolysis }-1 \text { day }\end{array}$ & $\$ 412.47$ & $\$ 232.40$ & $\$ 433.64$ & $\$ 238.94$ & $\$ 433.98$ & $\$ 240.99$ & $0.1 \%$ & $0.9 \%$ \\
\hline 62268 & $\begin{array}{l}\text { Percutaneous aspiration, } \\
\text { spinal cord cyst or syrinx }\end{array}$ & $\$ 352.34$ & $\$ 259.24$ & $\$ 296.81$ & $\$ 260.05$ & NA & $\$ 261.75$ & NA & $0.7 \%$ \\
\hline 62269 & $\begin{array}{l}\text { Biopsy of spinal cord, } \\
\text { percutaneous needle }\end{array}$ & $\$ 379.18$ & $\$ 266.04$ & $\$ 313.49$ & $\$ 264.81$ & NA & $\$ 264.13$ & NA & $-0.3 \%$ \\
\hline 62270 & Spinal puncture, diagnostic & $\$ 155.27$ & $\$ 78.83$ & $\$ 156.91$ & $\$ 78.29$ & $\$ 158.27$ & $\$ 77.95$ & $0.9 \%$ & $-0.4 \%$ \\
\hline 62272 & $\begin{array}{l}\text { Spinal puncture, } \\
\text { therapeutic }\end{array}$ & $\$ 192.65$ & $\$ 84.94$ & $\$ 200.14$ & $\$ 85.09$ & $\$ 202.86$ & $\$ 84.41$ & $1.4 \%$ & $-0.8 \%$ \\
\hline 62273 & Epidural, blood patch & $\$ 167.50$ & $\$ 111.44$ & $\$ 172.91$ & $\$ 113.35$ & $\$ 174.61$ & $\$ 114.03$ & $1.0 \%$ & $0.6 \%$ \\
\hline 62280 & $\begin{array}{l}\begin{array}{l}\text { Subarachnoid neurolytic } \\
\text { injection }\end{array} \\
\end{array}$ & $\$ 323.46$ & $\$ 162.07$ & $\$ 335.61$ & $\$ 167.47$ & $\$ 340.38$ & $\$ 177.34$ & $1.4 \%$ & $5.9 \%$ \\
\hline 62281 & Neurolytic epidural, $\mathrm{C} / \mathrm{T}$ & $\$ 259.24$ & $\$ 149.84$ & $\$ 250.52$ & $\$ 152.83$ & $\$ 246.09$ & $\$ 160.66$ & $-1.8 \%$ & $5.1 \%$ \\
\hline 62282 & Neurolytic epidural, L/S & $\$ 293.22$ & $\$ 139.64$ & $\$ 295.45$ & $\$ 142.28$ & $\$ 286.60$ & $\$ 145.00$ & $-3.0 \%$ & $1.9 \%$ \\
\hline 62284 & $\begin{array}{l}\text { Injection procedure } \\
\text { myelography }\end{array}$ & $\$ 213.71$ & $\$ 87.32$ & $\$ 201.50$ & $\$ 85.77$ & $\$ 190.27$ & $\$ 84.75$ & $-5.6 \%$ & $-1.2 \%$ \\
\hline 62287 & Disc decompression & NA & $\$ 549.40$ & NA & $\$ 560.26$ & NA & $\$ 571.15$ & NA & $1.9 \%$ \\
\hline 62290 & \begin{tabular}{|l|} 
Discography each level: \\
lumbar \\
\end{tabular} & $\$ 331.95$ & $\$ 170.56$ & $\$ 344.12$ & $\$ 172.91$ & $\$ 340.72$ & $\$ 175.29$ & $-1.0 \%$ & $1.4 \%$ \\
\hline 62291 & Discography each level: $\mathrm{C} / \mathrm{T}$ & $\$ 313.26$ & $\$ 164.45$ & $\$ 326.76$ & $\$ 166.44$ & $\$ 320.63$ & $\$ 168.15$ & $-1.9 \%$ & $1.0 \%$ \\
\hline 62292 & Chemonucleolysis & NA & $\$ 544.30$ & NA & $\$ 572.51$ & NA & $\$ 605.53$ & NA & $5.8 \%$ \\
\hline 62310 & \begin{tabular}{|l|} 
Cervical epidural \\
\end{tabular} & $\$ 230.36$ & $\$ 103.29$ & $\$ 246.77$ & $\$ 107.22$ & $\$ 247.11$ & $\$ 109.94$ & $0.1 \%$ & $2.5 \%$ \\
\hline 62311 & \begin{tabular}{|l|} 
Lumbar epidural \\
\end{tabular} & $\$ 197.74$ & $\$ 84.94$ & $\$ 208.99$ & $\$ 87.82$ & $\$ 206.95$ & $\$ 89.52$ & $-1.0 \%$ & $1.9 \%$ \\
\hline 62318 & $\begin{array}{l}\text { Epidural or subarachnoid, } \\
\text { catheterization, C/T }\end{array}$ & $\$ 236.82$ & $\$ 99.21$ & $\$ 251.20$ & $\$ 101.77$ & $\$ 235.20$ & $\$ 99.73$ & $-6.4 \%$ & $-2.0 \%$ \\
\hline 62319 & $\begin{array}{l}\text { Catheterization, epidural, } \\
\text { L/S }\end{array}$ & $\$ 188.91$ & $\$ 94.11$ & $\$ 185.16$ & $\$ 97.01$ & $\$ 170.87$ & $\$ 96.67$ & $-7.7 \%$ & $-0.4 \%$ \\
\hline
\end{tabular}


Pain Physician:September/October 2012; 15:E615-E627

Table 1 (cont.). 2013 proposed physician payment rates.

\begin{tabular}{|c|c|c|c|c|c|c|c|c|c|}
\hline \multirow{2}{*}{ CPT } & \multirow{2}{*}{ Description } & \multicolumn{2}{|c|}{$2011(\mathrm{CF}=\$ 33.9764)$} & \multicolumn{2}{|c|}{$2012(C F=\$ 34.0376)$} & \multicolumn{2}{|c|}{$\begin{array}{l}2013 \text { Proposed without } \\
\text { cut }(C F=\$ 34.0376)\end{array}$} & \multicolumn{2}{|c|}{ \% change from 2012} \\
\hline & & $\begin{array}{l}\text { Non-Facility } \\
\text { (Office) }\end{array}$ & $\begin{array}{c}\text { Facility } \\
\text { (ASC/Hospital) }\end{array}$ & $\begin{array}{l}\text { Non-Facility } \\
\text { (Office) }\end{array}$ & $\begin{array}{l}\text { Facility } \\
\text { (ASCl } \\
\text { Hospital) } \\
\end{array}$ & $\begin{array}{l}\text { Non-Facility } \\
\text { (Office) }\end{array}$ & $\begin{array}{c}\text { Facility } \\
\text { (ASC/Hospital) }\end{array}$ & $\begin{array}{l}\text { Non-Facility } \\
\text { (Office) }\end{array}$ & $\begin{array}{c}\text { Facility } \\
\text { (ASCl } \\
\text { Hospital) }\end{array}$ \\
\hline 62350 & \begin{tabular}{l|} 
Tunneled intrathecal or \\
epidural catheter for \\
long-term medication \\
administration via an external \\
pump or implantable \\
reservoir; w/o laminectomy
\end{tabular} & NA & $\$ 389.71$ & NA & $\$ 398.92$ & NA & $\$ 407.43$ & NA & $2.1 \%$ \\
\hline 62355 & $\begin{array}{l}\text { Removal or previously } \\
\text { implanted intrathecal or } \\
\text { epidural catheter }\end{array}$ & NA & $\$ 294.24$ & NA & $\$ 261.07$ & NA & $\$ 266.85$ & NA & $2.2 \%$ \\
\hline 62360 & $\begin{array}{l}\text { Implant or replacement } \\
\text { of device for intrathecal } \\
\text { or epidural drug infusion; } \\
\text { subcutaneous reservoir }\end{array}$ & NA & $\$ 300.69$ & NA & $\$ 309.40$ & NA & $\$ 314.85$ & NA & $1.8 \%$ \\
\hline 62361 & $\begin{array}{l}\text { Implantation or } \\
\text { replacement of device for } \\
\text { epidural drug infusion; } \\
\text { non-programmable pump }\end{array}$ & NA & $\$ 388.01$ & NA & $\$ 350.25$ & NA & $\$ 332.55$ & NA & $-5.1 \%$ \\
\hline 62362 & Implant spine infusion pump & NA & $\$ 406.70$ & NA & $\$ 389.05$ & NA & $\$ 393.47$ & NA & $1.1 \%$ \\
\hline 62365 & $\begin{array}{l}\text { Remove spine infusion } \\
\text { device }\end{array}$ & NA & $\$ 324.13$ & NA & $\$ 294.43$ & NA & $\$ 297.15$ & NA & $0.9 \%$ \\
\hline 62367 & $\begin{array}{l}\text { Electronic analysis of } \\
\text { programmable pump }\end{array}$ & $\$ 40.09$ & $\$ 24.46$ & $\$ 40.85$ & $\$ 24.85$ & $\$ 41.87$ & $\$ 25.53$ & $2.5 \%$ & $2.7 \%$ \\
\hline 62368 & $\begin{array}{l}\text { Electronic analysis of } \\
\text { programmable pump with } \\
\text { reprogramming }\end{array}$ & $\$ 57.76$ & $\$ 38.05$ & $\$ 55.14$ & $\$ 34.04$ & $\$ 56.50$ & $\$ 34.72$ & $2.5 \%$ & $2.0 \%$ \\
\hline 63650 & $\begin{array}{l}\text { Implant neuroelectrodes } \\
\text { (NA=National price is Not } \\
\text { Available) }\end{array}$ & NA & $\$ 414.85$ & NA & $\$ 427.17$ & NA & $\$ 437.04$ & NA & $2.3 \%$ \\
\hline 63655 & $\begin{array}{l}\text { Implant neuroelectrodes } \\
\text { (NA=National price is Not } \\
\text { Available) }\end{array}$ & NA & $\$ 855.53$ & NA & $\$ 832.90$ & NA & $\$ 834.60$ & NA & $0.2 \%$ \\
\hline 63661 & $\begin{array}{l}\begin{array}{l}\text { Remove spine electrode } \\
\text { percutaneous array(s) }\end{array} \\
\end{array}$ & $\$ 597.64$ & $\$ 330.25$ & $\$ 601.44$ & $\$ 325.40$ & $\$ 588.85$ & $\$ 325.40$ & $-2.1 \%$ & $0.0 \%$ \\
\hline 63662 & $\begin{array}{l}\text { Remove spine electrode } \\
\text { plate }\end{array}$ & NA & $\$ 716.56$ & NA & $\$ 785.93$ & NA & $\$ 780.14$ & NA & $-0.7 \%$ \\
\hline 63663 & $\begin{array}{l}\begin{array}{l}\text { Remove spine electrode } \\
\text { percutaneous array(s) }\end{array} \\
\end{array}$ & $\$ 853.83$ & $\$ 479.41$ & $\$ 855.36$ & $\$ 474.82$ & $\$ 830.52$ & $\$ 473.12$ & $-2.9 \%$ & $-0.4 \%$ \\
\hline 63664 & Remove spine eltrd plate & NA & $\$ 745.10$ & NA & $\$ 804.99$ & NA & $\$ 800.90$ & NA & $-0.5 \%$ \\
\hline 63685 & Implant neuroreceiver & NA & $\$ 396.50$ & NA & $\$ 363.18$ & NA & $\$ 368.29$ & NA & $1.4 \%$ \\
\hline 63688 & $\begin{array}{l}\text { Revise/remove } \\
\text { neuroreceiver }\end{array}$ & NA & $\$ 358.45$ & NA & $\$ 367.95$ & NA & $\$ 372.71$ & NA & $1.3 \%$ \\
\hline 64400 & $\begin{array}{l}\text { Injection, anesthetic agent; } \\
\text { Trigeminal nerve, any } \\
\text { division or branch }\end{array}$ & $\$ 112.80$ & $\$ 66.59$ & $\$ 118.11$ & $\$ 68.08$ & $\$ 122.88$ & $\$ 69.10$ & $4.0 \%$ & $1.5 \%$ \\
\hline 64402 & Facial nerve & $\$ 112.46$ & $\$ 72.71$ & $\$ 118.45$ & $\$ 74.88$ & $\$ 122.54$ & $\$ 76.24$ & $3.4 \%$ & $1.8 \%$ \\
\hline 64405 & Greater occipital nerve & $\$ 112.46$ & $\$ 79.50$ & $\$ 97.01$ & $\$ 61.95$ & $\$ 99.73$ & $\$ 62.63$ & $2.8 \%$ & $1.1 \%$ \\
\hline 64408 & Vagus nerve & $\$ 122.65$ & $\$ 90.72$ & $\$ 107.22$ & $\$ 78.63$ & $\$ 98.03$ & $\$ 72.84$ & $-8.6 \%$ & $-7.4 \%$ \\
\hline 64410 & Phrenic nerve & $\$ 148.14$ & $\$ 83.58$ & $\$ 152.15$ & $\$ 87.48$ & $\$ 126.62$ & $\$ 75.22$ & $-16.8 \%$ & $-14.0 \%$ \\
\hline 64412 & Spinal accessory nerve & $\$ 149.16$ & $\$ 74.07$ & $\$ 148.74$ & $\$ 74.20$ & $\$ 139.21$ & $\$ 72.84$ & $-6.4 \%$ & $-1.8 \%$ \\
\hline 64413 & Cervical plexus & $\$ 118.58$ & $\$ 78.83$ & $\$ 123.56$ & $\$ 80.33$ & $\$ 124.92$ & $\$ 80.67$ & $1.1 \%$ & $0.4 \%$ \\
\hline 64415 & Brachial plexus & $\$ 122.32$ & $\$ 67.27$ & $\$ 124.24$ & $\$ 66.71$ & $\$ 116.75$ & $\$ 63.99$ & $-6.0 \%$ & $-4.1 \%$ \\
\hline 64417 & Axillary nerve & $\$ 128.43$ & $\$ 69.65$ & $\$ 133.09$ & $\$ 71.14$ & $\$ 127.30$ & $\$ 69.10$ & $-4.3 \%$ & $-2.9 \%$ \\
\hline 64418 & Suprascapular nerve & $\$ 136.92$ & $\$ 73.39$ & $\$ 138.87$ & $\$ 74.20$ & $\$ 141.26$ & $\$ 74.88$ & $1.7 \%$ & $0.9 \%$ \\
\hline 64420 & Intercostal, single & $\$ 135.91$ & $\$ 66.59$ & $\$ 125.94$ & $\$ 68.08$ & $\$ 115.05$ & $\$ 69.44$ & $-8.6 \%$ & $2.0 \%$ \\
\hline 64421 & $\begin{array}{l}\text { Intercostal, multiple, } \\
\text { regional block }\end{array}$ & $\$ 195.36$ & $\$ 91.74$ & $\$ 177.68$ & $\$ 94.28$ & $\$ 155.89$ & $\$ 95.31$ & $-12.3 \%$ & $1.1 \%$ \\
\hline 64425 & Ilioinguinal, lliohypogastric & $\$ 130.47$ & $\$ 94.11$ & $\$ 134.45$ & $\$ 95.31$ & $\$ 136.15$ & $\$ 95.65$ & $1.3 \%$ & $0.4 \%$ \\
\hline 64445 & Sciatic nerve & $\$ 133.53$ & $\$ 75.43$ & $\$ 136.15$ & $\$ 74.20$ & $\$ 135.13$ & $\$ 71.48$ & $-0.7 \%$ & $-3.7 \%$ \\
\hline 64450 & $\begin{array}{l}\text { Other peripheral nerve or } \\
\text { branch }\end{array}$ & $\$ 102.27$ & $\$ 68.63$ & $\$ 105.52$ & $\$ 69.10$ & $\$ 108.92$ & $\$ 70.46$ & $3.2 \%$ & $2.0 \%$ \\
\hline 64479 & $\begin{array}{l}\text { Cervical transforaminal } \\
\text { epidural injections }\end{array}$ & $\$ 265.36$ & $\$ 131.15$ & $\$ 260.73$ & $\$ 134.11$ & $\$ 243.71$ & $\$ 136.15$ & $-6.5 \%$ & $1.5 \%$ \\
\hline
\end{tabular}


Proposed Physician Payment Schedule for 2013

Table 1 (cont.). 2013 proposed physician payment rates.

\begin{tabular}{|c|c|c|c|c|c|c|c|c|c|}
\hline \multirow{2}{*}{ CPT } & \multirow{2}{*}{ Description } & \multicolumn{2}{|c|}{$2011(\mathrm{CF}=\$ 33.9764)$} & \multicolumn{2}{|c|}{$2012(C F=\$ 34.0376)$} & \multicolumn{2}{|c|}{\begin{tabular}{|c|}
2013 Proposed without \\
cut $(C F=\$ 34.0376)$
\end{tabular}} & \multicolumn{2}{|c|}{ \% change from 2012} \\
\hline & & $\begin{array}{l}\text { Non-Facility } \\
\text { (Office) }\end{array}$ & $\begin{array}{c}\text { Facility } \\
\text { (ASC/Hospital) }\end{array}$ & $\begin{array}{l}\text { Non-Facility } \\
\text { (Office) }\end{array}$ & $\begin{array}{c}\text { Facility } \\
\text { (ASCI } \\
\text { Hospital) } \\
\end{array}$ & $\begin{array}{l}\text { Non-Facility } \\
\text { (Office) }\end{array}$ & $\begin{array}{c}\text { Facility } \\
\text { (ASC/Hospital) }\end{array}$ & $\begin{array}{l}\text { Non-Facility } \\
\text { (Office) }\end{array}$ & $\begin{array}{l}\text { Facility } \\
\text { (ASCl } \\
\text { Hospital) } \\
\end{array}$ \\
\hline 64480 & $\begin{array}{l}\text { Cervical transforaminal } \\
\text { epidural injections add-on }\end{array}$ & $\$ 126.39$ & $\$ 66.93$ & $\$ 124.92$ & $\$ 66.37$ & $\$ 116.41$ & $\$ 66.03$ & $-6.8 \%$ & $-0.5 \%$ \\
\hline 64483 & $\begin{array}{l}\text { Lumbar/sacral } \\
\text { transforaminal epidural } \\
\text { injections }\end{array}$ & $\$ 240.21$ & $\$ 102.61$ & $\$ 242.01$ & $\$ 111.98$ & $\$ 226.69$ & $\$ 114.03$ & $-6.3 \%$ & $1.8 \%$ \\
\hline 64484 & \begin{tabular}{|l|} 
Lumbar/sacral \\
transforaminal epidural \\
injections add-on \\
\end{tabular} & $\$ 106.35$ & $\$ 53.00$ & $\$ 100.07$ & $\$ 52.76$ & $\$ 89.52$ & $\$ 52.76$ & $-10.5 \%$ & $0.0 \%$ \\
\hline 64490 & $\begin{array}{l}\text { Cervical and thoracic facet } \\
\text { joint injections, 1st Level } \\
\text { (Old 64470) }\end{array}$ & $\$ 196.38$ & $\$ 111.44$ & $\$ 202.18$ & $\$ 110.96$ & $\$ 198.78$ & $\$ 109.94$ & $-1.7 \%$ & $-0.9 \%$ \\
\hline 64491 & $\begin{array}{l}\text { Cervical and thoracic facet } \\
\text { joint injections, 2nd Level } \\
\text { (Old 64472) }\end{array}$ & $\$ 97.17$ & $\$ 62.86$ & $\$ 98.37$ & $\$ 61.95$ & $\$ 96.33$ & $\$ 61.61$ & $-2.1 \%$ & $-0.5 \%$ \\
\hline 64492 & $\begin{array}{l}\text { Cervical and thoracic facet } \\
\text { joint injections, 3rd Level ( } \\
\text { New Code - Old 64472) }\end{array}$ & $\$ 98.19$ & $\$ 63.88$ & $\$ 99.05$ & $\$ 62.63$ & $\$ 97.01$ & $\$ 62.29$ & $-2.1 \%$ & $-0.5 \%$ \\
\hline 64493 & $\begin{array}{l}\text { Paravertebral facet joint or } \\
\text { facet joint nerve; lumbar/ } \\
\text { sacral, 1st Level (Old } \\
\text { 64475) }\end{array}$ & $\$ 174.98$ & $\$ 93.77$ & $\$ 181.08$ & $\$ 93.26$ & $\$ 178.70$ & $\$ 92.58$ & $-1.3 \%$ & $-0.7 \%$ \\
\hline 64494 & $\begin{array}{l}\text { Paravertebral facet joint or } \\
\text { facet joint nerve; lumbar/ } \\
\text { sacral, 2nd Level (Old } \\
\text { 64476) }\end{array}$ & $\$ 87.66$ & $\$ 53.34$ & $\$ 89.86$ & $\$ 52.42$ & $\$ 88.16$ & $\$ 52.08$ & $-1.9 \%$ & $-0.6 \%$ \\
\hline 64495 & $\begin{array}{l}\text { Paravertebral facet joint or } \\
\text { facet joint nerve; lumbar/ } \\
\text { sacral, 3rd Level (New } \\
\text { Code - Old 64476) }\end{array}$ & $\$ 89.02$ & $\$ 54.02$ & $\$ 90.54$ & $\$ 53.44$ & $\$ 88.50$ & $\$ 53.10$ & $-2.3 \%$ & $-0.6 \%$ \\
\hline 64505 & $\begin{array}{l}\text { Injection, anesthetic agent; } \\
\text { sphenopalatine ganglion }\end{array}$ & $\$ 97.85$ & $\$ 81.88$ & $\$ 100.41$ & $\$ 83.73$ & $\$ 103.47$ & $\$ 86.12$ & $3.1 \%$ & $2.8 \%$ \\
\hline 64508 & $\begin{array}{l}\text { Injection, anesthetic agent; } \\
\text { Carotid sinus (separate } \\
\text { procedure) }\end{array}$ & $\$ 104.31$ & $\$ 74.75$ & $\$ 86.12$ & $\$ 76.93$ & $\$ 66.37$ & $\$ 78.97$ & $-22.9 \%$ & $2.7 \%$ \\
\hline 64510 & $\begin{array}{l}\text { Injection, anesthetic agent; } \\
\text { Stellate ganglion (cervical } \\
\text { sympathetic) }\end{array}$ & $\$ 134.89$ & $\$ 69.65$ & $\$ 135.13$ & $\$ 72.50$ & $\$ 130.36$ & $\$ 74.54$ & $-3.5 \%$ & $2.8 \%$ \\
\hline 64520 & $\begin{array}{l}\text { Injection, anesthetic } \\
\text { agent; lumbar or thoracic } \\
\text { (paravertebral sympathetic) }\end{array}$ & $\$ 190.95$ & $\$ 78.15$ & $\$ 197.76$ & $\$ 80.33$ & $\$ 189.93$ & $\$ 81.01$ & $-4.0 \%$ & $0.8 \%$ \\
\hline 64530 & $\begin{array}{l}\text { Injection, anesthetic } \\
\text { agent; celiac plexus, with } \\
\text { or without radiologic } \\
\text { monitoring }\end{array}$ & $\$ 193.33$ & $\$ 90.38$ & $\$ 201.50$ & $\$ 92.92$ & $\$ 197.76$ & $\$ 94.62$ & $-1.9 \%$ & $1.8 \%$ \\
\hline 64600 & $\begin{array}{l}\text { Destruction by neurolytic } \\
\text { agent, trigeminal nerve; } \\
\text { supraorbital, infraorbital, } \\
\text { mental, or inferior alveolar } \\
\text { branch }\end{array}$ & $\$ 410.10$ & $\$ 219.83$ & $\$ 410.49$ & $\$ 219.88$ & $\$ 396.54$ & $\$ 219.54$ & $-3.4 \%$ & $-0.2 \%$ \\
\hline 64605 & $\begin{array}{l}\text { Destruction by neurolytic } \\
\text { agent, trigeminal nerve; } \\
\text { second and third division } \\
\text { branches at foramen ovale }\end{array}$ & $\$ 646.91$ & $\$ 340.10$ & $\$ 587.15$ & $\$ 336.63$ & $\$ 558.56$ & $\$ 341.06$ & $-4.9 \%$ & $1.3 \%$ \\
\hline 64610 & $\begin{array}{l}\text { Destruction by neurolytic } \\
\text { agent, trigeminal nerve; } \\
\text { second and third division } \\
\text { branches at foramen ovale } \\
\text { under radiologic }\end{array}$ & $\$ 727.77$ & $\$ 486.88$ & $\$ 755.29$ & $\$ 488.78$ & $\$ 744.74$ & $\$ 486.40$ & $-1.4 \%$ & $-0.5 \%$ \\
\hline 64612 & $\begin{array}{l}\text { Chemodenervation of } \\
\text { muscle(s); muscle(s) } \\
\text { innervated by facial nerve } \\
\text { (eg, for blepharospasm, } \\
\text { hemifacial spasm) }\end{array}$ & $\$ 171.24$ & $\$ 155.95$ & $\$ 174.61$ & $\$ 160.32$ & $\$ 177.34$ & $\$ 164.74$ & $1.6 \%$ & $2.8 \%$ \\
\hline 64613 & $\begin{array}{l}\text { Chemodenervation of } \\
\text { muscle(s); neck muscle(s) } \\
\text { (eg, for spasmodic } \\
\text { torticollis, spasmodic } \\
\text { dysphonia) }\end{array}$ & $\$ 164.11$ & $\$ 145.42$ & $\$ 166.10$ & $\$ 149.77$ & $\$ 168.15$ & $\$ 154.19$ & $1.2 \%$ & $3.0 \%$ \\
\hline
\end{tabular}


Pain Physician:September/October 2012; 15:E615-E627

Table 1 (cont.). 2013 proposed physician payment rates.

\begin{tabular}{|c|c|c|c|c|c|c|c|c|c|}
\hline \multirow{2}{*}{ CPT } & \multirow{2}{*}{ Description } & \multicolumn{2}{|c|}{$2011(\mathrm{CF}=\$ 33.9764)$} & \multicolumn{2}{|c|}{$2012(C F=\$ 34.0376)$} & \multicolumn{2}{|c|}{$\begin{array}{l}2013 \text { Proposed without } \\
\text { cut }(C F=\$ 34.0376)\end{array}$} & \multicolumn{2}{|c|}{ \% change from 2012} \\
\hline & & $\begin{array}{l}\text { Non-Facility } \\
\text { (Office) }\end{array}$ & $\begin{array}{c}\text { Facility } \\
\text { (ASC/Hospital) }\end{array}$ & $\begin{array}{l}\text { Non-Facility } \\
\text { (Office) }\end{array}$ & $\begin{array}{l}\text { Facility } \\
\text { (ASC/ } \\
\text { Hospital) }\end{array}$ & $\begin{array}{l}\text { Non-Facility } \\
\text { (Office) }\end{array}$ & $\begin{array}{c}\text { Facility } \\
\text { (ASC/Hospital) }\end{array}$ & $\begin{array}{l}\text { Non-Facility } \\
\text { (Office) }\end{array}$ & $\begin{array}{l}\text { Facility } \\
\text { (ASCl } \\
\text { Hospital) }\end{array}$ \\
\hline 64614 & $\begin{array}{l}\text { Chemodenervation of } \\
\text { muscle(s); extremity(s) and/ } \\
\text { or trunk muscle(s) (eg, for } \\
\text { dystonia, cerebral palsy, } \\
\text { multiple sclerosis) }\end{array}$ & $\$ 174.98$ & $\$ 151.87$ & $\$ 177.68$ & $\$ 156.23$ & $\$ 179.38$ & $\$ 159.98$ & $1.0 \%$ & $2.4 \%$ \\
\hline 64620 & $\begin{array}{l}\text { Destruction by neurolytic } \\
\text { agent, intercostal nerve }\end{array}$ & $\$ 238.85$ & $\$ 169.54$ & $\$ 225.33$ & $\$ 173.25$ & $\$ 210.01$ & $\$ 176.66$ & $-6.8 \%$ & $2.0 \%$ \\
\hline 64630 & $\begin{array}{l}\text { Destruction by neurolytic } \\
\text { agent; pudendal nerve }\end{array}$ & $\$ 225.26$ & $\$ 187.89$ & $\$ 230.43$ & $\$ 190.61$ & $\$ 213.08$ & $\$ 180.40$ & $-7.5 \%$ & $-5.4 \%$ \\
\hline 64633 & $\begin{array}{l}\text { Destruction by neurolytic } \\
\text { agent, paravertebral } \\
\text { facet joint nerve; cervical } \\
\text { or thoracic, single level } \\
(64626)\end{array}$ & $\$ 398.54$ & $\$ 247.69$ & $\$ 452.36$ & $\$ 235.54$ & $\$ 405.05$ & $\$ 220.56$ & $-10.5 \%$ & $-6.4 \%$ \\
\hline 64634 & $\begin{array}{l}\text { Destruction by neurolytic } \\
\text { agent, paravertebral facet } \\
\text { joint nerve; cervical or } \\
\text { thoracic, each additional } \\
\text { level (64627) }\end{array}$ & $\$ 170.90$ & $\$ 58.78$ & $\$ 207.29$ & $\$ 70.46$ & $\$ 183.46$ & $\$ 66.71$ & $-11.5 \%$ & $-5.3 \%$ \\
\hline 64635 & $\begin{array}{l}\text { Destruction by neurolytic } \\
\text { agent, paravertebral facet } \\
\text { joint nerve; lumbar or } \\
\text { sacral, single level (old } \\
64622 \text { ) }\end{array}$ & $\$ 335.01$ & $\$ 182.79$ & $\$ 444.53$ & $\$ 230.77$ & $\$ 397.90$ & $\$ 217.50$ & $-10.5 \%$ & $-5.8 \%$ \\
\hline 64636 & $\begin{array}{l}\text { Destruction by neurolytic } \\
\text { agent, paravertebral facet } \\
\text { joint nerve; lumbar or } \\
\text { sacral, each additional level } \\
(64623)\end{array}$ & $\$ 125.03$ & $\$ 49.95$ & $\$ 186.53$ & $\$ 61.27$ & $\$ 165.08$ & $\$ 58.54$ & $-11.5 \%$ & $-4.4 \%$ \\
\hline 64640 & $\begin{array}{l}\text { Destruction by neurolytic } \\
\text { agent; other peripheral } \\
\text { nerve or branch }\end{array}$ & $\$ 217.79$ & $\$ 168.18$ & $\$ 219.88$ & $\$ 169.85$ & $\$ 221.58$ & $\$ 172.23$ & $0.8 \%$ & $1.4 \%$ \\
\hline 64680 & $\begin{array}{l}\text { Destruction by neurolytic } \\
\text { agent, with or without } \\
\text { radiologic monitoring; } \\
\text { celiac plexus }\end{array}$ & $\$ 313.60$ & $\$ 163.09$ & $\$ 325.74$ & $\$ 166.44$ & $\$ 319.27$ & $\$ 169.85$ & $-2.0 \%$ & $2.0 \%$ \\
\hline
\end{tabular}

Based on 2012 Conversion Factor - NA - Not Applicable \% change - from 2012 - As of Aug 10, 2012

* 2013 Conversion Factor will be published by November 1, 2012 as part of the Calendar Year 2013 Physician Fee Schedule final rule, at present the rates are based on 2012 conversion factors.

Table 2. Calendar Year 2013 Physician Fee Schedule proposed rule estimated impact on total allowed charges by specialty*.

\begin{tabular}{|c|c|c|c|c|c|}
\hline (A) & \multicolumn{2}{|c|}{ (B) } & (C) & (D) & $(\mathbf{E})$ \\
\hline Specialty & \multicolumn{2}{|c|}{$\begin{array}{c}\text { Allowed Charges } \\
\text { (mil) }\end{array}$} & $\begin{array}{l}\text { Impact of Work and } \\
\text { MP RVU Changes }\end{array}$ & $\begin{array}{l}\text { Impact of PE } \\
\text { RVU Changes }\end{array}$ & $\begin{array}{c}\text { Combined } \\
\text { Impact }\end{array}$ \\
\hline TOTAL & $\$$ & 86,000 & $0 \%$ & $0 \%$ & $0 \%$ \\
\hline 01-ALLERGY/IMMUNOLOGY & $\$$ & 198 & $-1 \%$ & $1 \%$ & $0 \%$ \\
\hline 02-ANESTHESIOLOGY & $\$$ & 1,970 & $-1 \%$ & $-3 \%$ & $-3 \%$ \\
\hline 03-CARDIAC SURGERY & $\$$ & 366 & $-1 \%$ & $-2 \%$ & $-2 \%$ \\
\hline 04-CARDIOLOGY & $\$$ & 6,568 & $-1 \%$ & $-2 \%$ & $-3 \%$ \\
\hline 05-COLON AND RECTAL SURGERY & $\$$ & 153 & $-1 \%$ & $1 \%$ & $1 \%$ \\
\hline 06-CRITICAL CARE & $\$$ & 261 & $-1 \%$ & $0 \%$ & $0 \%$ \\
\hline 07-DERMATOLOGY & $\$$ & 3,008 & $-1 \%$ & $0 \%$ & $0 \%$ \\
\hline 08-EMERGENCY MEDICINE & $\$$ & 2,819 & $-1 \%$ & $0 \%$ & $-1 \%$ \\
\hline 09-ENDOCRINOLOGY & $\$$ & 434 & $-1 \%$ & $1 \%$ & $1 \%$ \\
\hline 10-FAMILY PRACTICE & $\$$ & 5,879 & $3 \%$ & $4 \%$ & $7 \%$ \\
\hline 11-GASTROOENTEROLOGY & $\$$ & 1,885 & $-1 \%$ & $0 \%$ & $0 \%$ \\
\hline 12-GENERAL PRACTICE & $\$$ & 579 & $-1 \%$ & $1 \%$ & $0 \%$ \\
\hline 13-GENERAL SURGERY & $\$$ & 2,261 & $-1 \%$ & $0 \%$ & $0 \%$ \\
\hline 14-GERIATIRCS & $\$$ & 217 & $1 \%$ & $3 \%$ & $4 \%$ \\
\hline
\end{tabular}


Proposed Physician Payment Schedule for 2013

Table 2 (cont.). Calendar Year 2013 Physician Fee Schedule proposed rule estimated impact on total allowed charges by specialty*.

\begin{tabular}{|c|c|c|c|c|c|}
\hline (A) & & В) & (C) & (D) & $(\mathrm{E})$ \\
\hline Specialty & Allor & $\begin{array}{l}\text { d Charges } \\
\text { nil) }\end{array}$ & $\begin{array}{l}\text { Impact of Work and } \\
\text { MP RVU Changes }\end{array}$ & $\begin{array}{l}\text { Impact of PE } \\
\text { RVU Changes }\end{array}$ & $\begin{array}{c}\text { Combined } \\
\text { Impact }\end{array}$ \\
\hline 15-HAND SURGERY & $\$$ & 134 & $-1 \%$ & $0 \%$ & $0 \%$ \\
\hline 16-HEMATOLOGY ONCOLOGY & $\$$ & 1,900 & $-1 \%$ & $0 \%$ & $-1 \%$ \\
\hline 17-INFECTIOUS DISEASE & $\$$ & 623 & $-1 \%$ & $1 \%$ & $0 \%$ \\
\hline 18-INTERNAL MEDICINE & $\$$ & 11,058 & $2 \%$ & $3 \%$ & $5 \%$ \\
\hline 19-INTERVENTIONAL PAIN MGMT & $\$$ & 534 & $-1 \%$ & $0 \%$ & $-1 \%$ \\
\hline 20-INTERVENTIONAL RADIOLOGY & $\$$ & 203 & $-1 \%$ & $-2 \%$ & $-3 \%$ \\
\hline 21-MULTISPECIALTY CLINIC-OTHER PHY & $\$$ & 202 & $-1 \%$ & $-1 \%$ & $-1 \%$ \\
\hline 22-NEPGROLOGY & $\$$ & 2,065 & $-1 \%$ & $0 \%$ & $-1 \%$ \\
\hline 23-NEUROLOGY & $\$$ & 1,601 & $-1 \%$ & $2 \%$ & $1 \%$ \\
\hline 24-NEUROSURGERY & $\$$ & 681 & $-1 \%$ & $0 \%$ & $-1 \%$ \\
\hline 25-NUCLEAR MEDICINE & $\$$ & 49 & $-1 \%$ & $-3 \%$ & $-3 \%$ \\
\hline 26-OBSTETRICS/GYNECOLOGY & $\$$ & 698 & $-1 \%$ & $0 \%$ & $1 \%$ \\
\hline 27-OPHTHALMOLOGY & $\$$ & 5,621 & $-1 \%$ & $1 \%$ & $1 \%$ \\
\hline 28-ORTHOPEDIC SURGERY & $\$$ & 3,622 & $-1 \%$ & $0 \%$ & $-1 \%$ \\
\hline 29-OTOLARYNGOLOGY & $\$$ & 1,070 & $-1 \%$ & $1 \%$ & $0 \%$ \\
\hline 30-PATHOLOGY & $\$$ & 1,185 & $-1 \%$ & $-1 \%$ & $-2 \%$ \\
\hline 31-PEDIATRICS & $\$$ & 64 & $2 \%$ & $3 \%$ & $5 \%$ \\
\hline 32-PHYSICAL MEDICINE & $\$$ & 990 & $-1 \%$ & $1 \%$ & $1 \%$ \\
\hline 33-PLASTIC SURGERY & $\$$ & 351 & $-1 \%$ & $0 \%$ & $0 \%$ \\
\hline 34-PSYCHIATRY & $\$$ & 1,149 & $-1 \%$ & $0 \%$ & $0 \%$ \\
\hline 35-PULMONARY DISEASE & $\$$ & 1,691 & $-1 \%$ & $1 \%$ & $0 \%$ \\
\hline 36-RADIATION ONCOLOGY & $\$$ & 1,983 & $-1 \%$ & $-14 \%$ & $-14 \%$ \\
\hline 37-RADIOLOGY & $\$$ & 4,791 & $-1 \%$ & $-3 \%$ & $-4 \%$ \\
\hline 38-RHEUMATOLOGY & $\$$ & 545 & $-1 \%$ & $0 \%$ & $0 \%$ \\
\hline 39-THORACIC SURGERY & $\$$ & 340 & $-1 \%$ & $-1 \%$ & $-2 \%$ \\
\hline 40-UROLOGY & $\$$ & 1,909 & $-1 \%$ & $-1 \%$ & $-2 \%$ \\
\hline 41-VASCULAR SURGERY & $\$$ & 882 & $-1 \%$ & $-2 \%$ & $-3 \%$ \\
\hline 42-AUDIOLOGIST & $\$$ & 57 & $-1 \%$ & $-4 \%$ & $-5 \%$ \\
\hline 43-CHIROPRACTOR & $\$$ & 738 & $-1 \%$ & $1 \%$ & $1 \%$ \\
\hline 44-CLINICAL PSYCHOLOGIST & $\$$ & 567 & $-1 \%$ & $-2 \%$ & $-3 \%$ \\
\hline 45-CLINICAL SOCIAL WORKER & $\$$ & 400 & $-1 \%$ & $-2 \%$ & $-3 \%$ \\
\hline 46-DIAGNOSTIC TESTING FACILITY & $\$$ & 875 & $-1 \%$ & $-7 \%$ & $-8 \%$ \\
\hline 47-INDEPENDENT LABORATORY & $\$$ & 1,064 & $-1 \%$ & $-1 \%$ & $-1 \%$ \\
\hline 48-NURSE ANES/ANES ASST & $\$$ & 1,142 & $-1 \%$ & $-3 \%$ & $-4 \%$ \\
\hline 49-NURSE PRACTITIONER & $\$$ & 1,606 & $1 \%$ & $3 \%$ & $5 \%$ \\
\hline 50-OPTOMETRY & $\$$ & 1,048 & $-1 \%$ & $2 \%$ & $1 \%$ \\
\hline 51-ORAL/MAXILLOFACIAL SURGERY & $\$$ & 44 & $-1 \%$ & $1 \%$ & $0 \%$ \\
\hline 52-PHYSICAL/OCCUPATIONAL THERAPY & $\$$ & 2,613 & $-1 \%$ & $3 \%$ & $3 \%$ \\
\hline 53-PHYSICIAN ASSISTANT & $\$$ & 1,219 & $1 \%$ & $2 \%$ & $3 \%$ \\
\hline 54-PODIATRY & $\$$ & 1,898 & $-1 \%$ & $2 \%$ & $1 \%$ \\
\hline 55-PORTABLE X-RAY SUPPLIER & $\$$ & 104 & $-1 \%$ & $2 \%$ & $2 \%$ \\
\hline 56-RADIATION THERAPY CENTERS & $\$$ & 71 & $-1 \%$ & $-18 \%$ & $-19 \%$ \\
\hline 98-OTHER & $\$$ & 19 & $-1 \%$ & $1 \%$ & $0 \%$ \\
\hline
\end{tabular}

* Table shows only the proposed payment policy impact on Physician Fee Schedule services. We note that these impacts do not include the effects of 2013 . 
The second major issue relates to scope of practice (41-50). Scope of practice issues are based on the Obama administration's philosophy to reform health care regulations that it views as unnecessary(51). In particular, the Administration said, "the use of advanced practice nurse practitioners and physician assistants in lieu of higher paid physicians could provide immediate savings to hospitals" (51). In the new rules, CMS proposes to remove barriers to the work of physician extenders, for example, by not making them seek out a physician to cosign every order. However, it does not stop there.

The Obama Administration has been in the process of empowering nurses (8). At the same time, they have proposed allowing CRNAs to perform complex interventional pain management services. While we all recognize that midlevel providers are on every team and are essential to health care as long as they are working as part of the team, they should not be replacing physicians. The independent practice of CRNAs started with an opt out mechanism provided by CMS that was signed into law by President Clinton, whose mother was a nurse anesthetist (52). While the opt out was a good idea and was originally intended to help rural areas improve access to care, the rule was transformed into supporting any hospital that seeks to cut costs by allowing nurse anesthetists to provide independent anesthesia, which now is being translated to the independent practice of interventional pain management. Now, with the CMS chief, Marilyn Tavenner, being a nurse, nurses are employing tactics to remove physicians from the equation (42-46).

The Obama Administration rightfully has expressed concern about the impending shortage of physicians as a reason to allow for more latitude to advanced practice nurses. However, there is no one else to blame other than the Obama Administration for the projected shortage. The physician shortage may be alleviated by providing physicians a reprieve rather than replacing them with midlevel providers. The American Medical Association (AMA), American Society of Interventional Pain Physicians (ASIPP), and American Society of Anesthesiologists (ASA) have opposed this proposed regulation that would allow CRNAs to perform interventional procedures. (53-57)

With all the excitement about ObamaCare and its US Supreme Court survival, there are multiple proposals to reform or modernize Medicare. One such proposal is from Senator Ron Wyden (D-OR) and Representative Paul Ryan (R-WI). Their proposal is named "Bipartisan Options for the Future" (58). Wilensky (59) described that the notion that Democrats and Republicans agreed about certain aspects of Medicare might have seemed unthinkable. He hopes that the pairing of a liberal Democrat who has long worked on health care reforms and a fiscally conservative Republican primarily known for work on budget issues, now the Republican vice presidential candidate, suggests that it might be possible for the parties to reach a compromise on Medicare reform. It appears that there is a bipartisan agreement to change Medicare that might make it more efficient, effective, and fiscally sustainable, even if none of these changes are universally accepted by either party as desirable or even tolerable.

In conclusion, interventional pain management continues to face widespread challenges, more so than other specialties in the US health care system. The historic health care reform which was passed by Congress and signed into law by President Obama, and subsequently supported to a great extent by a Supreme Court decision, is affecting medicine drastically in the United States. Emanuel and Fuchs (60) have proposed to shorten medical training by $30 \%$ which in their opinion will equalize mid-level practitioners including nurse anesthetists and physician anesthesiologists including interventional pain physicians. An evolving specialty, interventional pain management is being encroached upon by many special interests, including now nurse anesthetists. As a result, our specialty will probably suffer the most in the next year and in the years to come.

\section{Acknowledgments}

The authors wish to thank Vidyasagar Pampati, MSc, for statistical assistance, Sekar Edem for assistance in the search of the literature, Tom Prigge, MA, for manuscript review, and Tonie M. Hatton and Diane E. Neihoff, transcriptionists, for their assistance in preparation of this manuscript. We would like to thank the editorial board of Pain Physician for review and criticism in improving the manuscript.

\section{Author Affiliation}

Dr. Manchikanti is Medical Director of the Pain Management Center of Paducah, Paducah, KY and Clinical Professor, Anesthesiology and Perioperative Medicine, University of Louisville, Louisville, KY. Dr. Singh is Medical Director, Spine Pain Diagnostics Associates, Niagara, WI. Dr. Caraway, St. Mary's Pain Relief Center, Huntington, West Virginia. Dr. Benyamin is the Medical Director, Millennium Pain Center, Bloomington, IL, Clinical Assistant Professor of Surgery, College of Medicine, University of Illinois, Urbana-Champaign, 
IL. Dr. Falco is Medical Director of Mid Atlantic Spine \& Pain Physicians, DE; Director, Pain Medicine Fellowship Program, Temple University Hospital, Philadelphia, PA and Associate Professor, Department of PM\&R, Temple University Medical School, Philadelphia, PA Dr. Hirsch is Chief of Minimally Invasive Spine Surgery, Depts. of Radiology and Neurosurgery, Massachusetts General Hospital and Associate Professor of Radiology, Harvard Medical School, Boston, MA.

\section{Disclosures}

Dr. Caraway is a consultant for Medtronic, Inc., Spinal Modulation, Inc., and Vertos, Inc.

Dr. Falco is a consultant for St. Jude Medical Inc. and Joimax Inc.

Dr. Benyamin is a consultant with Bioness and Nevro, serves on the advisory boards of Vertos Medical and Nuvo Pharma, teaches/lectures for Vertos Medical, Boston Scientific, Neurotherm, and Bioness, and receives research/grants from Alfred Mann Foundation, Teknon Foundation, Spinal Restoration, Inc., Bioness, Boston Scientific, Vertos Medical, Medtronic, Kimberly Clarke, Epimed, BioDelivery Sciences International, Inc., Theravance, Mundipharma Research, Cephalon/Teva, AstraZeneca, and Purdue Pharma, LP.

Dr. Hirsch has received fees from CareFusion in the past 12 months. He participated in an Aetrium focus group and received compensation.

\section{References}

1. Department of Health and Human Services, Centers for Medicare \& Medicaid Services. 42 CFR Parts 410, 414, 415, 421, 423, 425, 486, and 495. Medicare Program; Revisions to Payment Policies Under the Physician Fee Schedule, DME Face-to-Face Encounters, Elimination of the Requirement for Termination of Non-Random Prepayment Complex Medical Review and Other Revisions to Part B for CY 2013. Proposed Rule. 2012.

2. Manchikanti L, Singh V, Caraway DL, Benyamin RM, Falco FJE, Hirsch JA. Physician payment outlook for 2012: Déjà Vu. Pain Physician 2012; 15:E27-E52.

3. Manchikanti L, Hirsch JA. Physician payment outlook for 2012. J Neurolntervent Surg 2012; Online First 20 June 2012.

4. Manchikanti L, Singh V, Caraway DL, Benyamin RM, Hirsch JA. Medicare physician payment systems: Impact of 2011 schedule on interventional pain management. Pain Physician 2011; 14: $E_{5}-E_{33}$.

5. Manchikanti L, Hirsch JA. Medicare physician payment rules for 2011: A primer for the neurointerventionalist. J Neurointervent Surg 2011; 3:399-402.

6. Manchikanti L, Parr AT, Singh V, Fellows B. Ambulatory surgery centers and interventional techniques: A look at long-term survival. Pain Physician 2011; 14:E177-E215.

7. Manchikanti L, Singh V, Hirsch JA. Saga of payment systems of ambulatory surgery centers for interventional techniques: An update. Pain Physician 2012; 15:109-130.

8. Manchikanti L, Caraway DL, Falco FJE,
Benyamin RM, Hansen $\mathrm{H}$, Hirsch JA. CMS proposal for interventional pain management by nurse anesthetists: Evidence by proclamation. Pain Physician 2012;

9. Medicare's Payments to Physicians: The Budgetary Impact of Alternative Policies," CBO, June 16, 2011.

10. The Budget Control Act of 2011 (Debt Ceiling Deal). OMB Watch. www.ombwatch.org/files/budget/debtceilingfaq. pdf

11. Hahn J, Mulvey J. Congressional Research Service (CRS) Report for Congress. Medicare Physician Payment Updates and the Sustainable Growth Rate (SGR) System. December 27, 2011.

http://www.ascrs.org/download/ gov/Medicare\%2oPhysician\%2oPayment\%2oUpdates\%2oand\%20the\%20 Sustainable\%20G rowth\%20Rate\%20 SGR\%2oSystem.pdf

12. Manchikanti L, Falco FJE, Hirsch JA. Necessity and implications of ICD-10: Facts and fallacies. Pain Physician 2011; 14:E405-E425.

13. Manchikanti L, Falco FJE, Hirsch JA. Ready or not! Here comes ICD-10. J Neurointerv Surg 2011; Published Online First: October 26, 2011.

14. 2012 ICD-10-CM: International Classification of Diseases, 1oth Revision - Clinical Modification, Professional ed. Saunders/ Elsevier, St. Louis, 2012.

15. Centers for Disease Control and Prevention (CDC). Adult use of prescription opioid pain medications - Utah, 2008. MMWR Morb Mortal Wkly Rep 2010;
59:153-157.

16. Centers for Disease Control and Prevention. CDC grand rounds: Prescription drug overdoses - a U.S. epidemic. MMWR Morb Mortal Wkly Rep 2012; 61:10-13.

17. Manchikanti L, Falco FJE, Benyamin RM, Caraway DL, Helm II S, Wargo BW, Hansen H, Parr AT, Singh V, Hirsch JA. Assessment of infection control practices for interventional techniques: A best evidence synthesis of safe injection practices and use of single-dose medication vials. Pain Physician 2012; 15:E573E614.

18. Manchikanti L, Malla Y, Wargo BW, Fellows B. Infection control practices (safe injection and medication vial utilization) for interventional techniques: Are they based on relative risk management or evidence? Pain Physician 2011; 14:425434.

19. Letter to Kathleen Sebelius, Secretary of Health and Human Services from American Society of Interventional Pain Physicians RE: Critical Shortage of Drugs and Increasing Anxiety and Expenses: A Request for Reduction of the Regulatory Burden on Physicians, Including the Use of Single Dose Vials for Infection Control, Implementation of ICD10, and EMR Regulation, November 18, 2011.

20. Letter to Elizabeth L. Skillen, PhD, Associate Director for Policy, Centers for Disease Control and Prevention, from American Society of Interventional Pain Physicians RE: CDC's Position on Protecting Patients Against Preventable 
Harm for Improper Use of Single-Dose/ Single-Use Vials, May 8, 2012.

21. Letter to Laxmaiah Manchikanti, MD from Marilyn Tavenner, Acting Administrator of Centers for Medicare and Medicaid Services (CMS). RE: Single-dose vials for Infection Control, ICD-10, and EHR. July 12, 2012.

22. Letter to Kathleen Sebelius, Secretary of Health and Human Services from Members of Congress, United States Representative RE: Single dose vials, March 15, 2012.

23. Letter to The Honorable Ed Whitfield and U.S. House of Representatives from Kathleen Sebelius RE: Single-Dose vial (SDV) policy, May 10, 2012.

24. Letter to Kathleen Sebelius, Secretary of Health and Human Services from Cliff Stearns, United States Representative RE: Single dose vials, May 15, 2012.

25. Letter to Kathleen Sebelius, Secretary of Health and Human Services from Rand Paul and David Vitter, United States Senators RE: Single dose vials, June 7, 2012.

26. Public Law No: 111-148: H.R. 3590. Patient Protection and Affordable Care Act. March 23, 2010.

27. Manchikanti L, Caraway DL, Parr AT, Fellows B, Hirsch JA. Patient Protection and Affordable Care Act of 2010: Reforming health care reform for the new decade. Pain Physician 2011; 14:E35-E67.

28. Manchikanti L, Hirsch JA. Patient Protection and Affordable Care Act of 2010: A Primer for Neurolnterventionalists. ] Neurointervent Surg 2012; 4:141-146.

29. Manchikanti L, Singh V, Boswell MV. Interventional pain management at crossroads: The perfect storm brewing for a new decade of challenges. Pain Physician 2010; 13:Eı11-E140.

30. Benyamin RM, Datta S, Falco FJE. A perfect storm in interventional pain management: Regulated, but unbalanced. Pain Physician 2010; 13:109-116.

31. S. 1213: Patient-Centered Outcomes Research Act of 2009 (Introduced in Senate). June 9, 2009.

32. Manchikanti L, Falco FJ, Benyamin RM, Helm S 2nd, Parr AT, Hirsch JA. The impact of comparative effectiveness research on interventional pain management: Evolution from Medicare Modernization Act to Patient Protection and Affordable Care Act and the PatientCentered Outcomes Research Institute. Pain Physician 2011; 14:E249-E282.

33. Manchikanti L, Helm II S, Hirsch JA. The evolution of the Patient-Centered Out- come Research Institute. J Neurointervent Surg 2012; 4:157-162.

34. Manchikanti L, Falco FJE, Boswell MV Hirsch JA. Facts, fallacies, and politics of comparative effectiveness research: Part 1. Basic considerations. Pain Physician 2010; 13:E23-E54.

35. Manchikanti L, Falco FJE, Boswell MV, Hirsch JA. Facts, fallacies, and politics of comparative effectiveness research: Part 2. Implications for interventional pain management. Pain Physician 2010; 13:E55-E79.

36. H.R. 452. Medicare Decisions Accountability Act of 2011. January 25, 2011.

37. Manchikanti L, Falco FJE, Singh V, Benyamin RM, Hirsch JA. The Independent Payment Advisory Board. Pain Physician 2011; 14:E313-E342.

38. Manchikanti L, Hirsch JA. The Independent Payment Advisory Board: Impact on neurointerventionalists. J Neurointerv Surg 2011; Published Online First: October 11, 2011.

39. Medicare Payment Advisory Commission. Report to the Congress. Moving forward from the sustainable growth rate (SGR) system. October 14 2011. http://www.medpac.gov/documents/10142011_MedPAC_SGR_letter. pdf

40. Mitchell J, Bendavid N. Tax Bill Passed by Congress Broadens Jobless Program. The Wall Street Journal, February 18, 2012

http://online.wsj.com/article/SB1000142 4052970204880404577229180280277616 . html

41. Department of Health and Human Services. Office of the Inspector General. Prevalence and Qualifications of Nonphysicians Who Performed Medicare Physician Services. August 2009 http://oig.hhs.gov/ oei/reports/oei-09-06-00430.pdf

42. Institute of Medicine (IOM). The Future of Nursing: Leading Change, Advancing Health. The National Academies Press, Washington, DC. October 5, 2010

43. Clavelle JT. Implementing Institute of Medicine future of nursing recommendations: A model for transforming nurse practitioner privileges. J Nurs Adm 2012; 42:404-407.

44. Brooten D, Youngblut JM, Hannan J, Guido-Sanz F. The impact of interprofessional collaboration on the effectiveness, significance, and future of advanced practice registered nurses. Nurs Clin North Am 2012; 47:283-294

45. Roberts A, Sutton JH. Scope of practice for nonsurgeons keeps expanding. Bull
Am Coll Surg 2001; 86:15-18.

46. Vaughn D. CMS proposes to pay CRNAs to perform chronic pain. Vaughn \& Associates, July 12, 2012.

47. Kuehn BM. IOM: Boost Nurses' role in health care. JAMA 2010; 304:2345-2346.

48. Carlson J. Fueling the turf battle. Institute of Medicine report pushes expanded scope for nurses; docs say study draws illogical conclusions. Mod Healthc 2010; 40:6-7, 16, 1.

49. Hudspeth R. Complex healthcare reg ulation: managing the continued challenge of competence. Nurs Adm Q 2008; 32:74-75.

50. Braverman AS. Power and medical practice. Pharos Alpha Omega Alpha Honor Med Soc 1999; 62:30-32.

51. Sibert KS. Unsupervised anesthesia care by a nurse anesthetist is a threat to patient safety. Physician, November 17, 2011.

www.kevinmd.com/blog/2011/11/unsupervised-anesthesia-care-nurse-anesthetist-threat-patient-safety.html

52. Department of Health and Human Services, Health Care Financing Administration. 42 CFR Parts 410 and 414 Medicare Program; Revisions to Payment Policies Under the Physician Fee Schedule for Calendar Year 2001; Final Rule. November 1, 2000.

53. Letter to Marilyn Tavenner, Acting Administrator of Centers for Medicare and Medicaid Services (CMS) from American Society of Interventional Pain Physician re 42 CFR Parts 410, 414, 415, 421, 423, 425, 486, and 495. CMS-1590-P. Medicare Program; Revisions to Payment Policies Under the Physician Fee Schedule, DME Face-to-Face Encounters, Elimination of the Requirement for Termination of Non-Random Prepayment Complex Medical Review and Other Revisions to Part B for CY 2013. August 31, 2012.

54. Letter to Kathleen Sebelius, Secretary of Department of Health and Human Services, and Marilyn Tavenner, Acting Administrator of Centers for Medicare and Medicaid Services (CMS) from American Society of Interventional Pain Physician re CRNAs and Interventional Pain Management. June 28, 2012.

55. Letter to Laxmaiah Manchikanti, MD, from Marilyn Tavenner, Acting Administrator of Centers for Medicare and Medicaid Services (CMS) re Certified Registered Nurse Anesthetists (CRNAs). August 13, 2012. 
56. Letter to Marilyn Tavenner, Acting Administrator of Centers for Medicare and Medicaid Services (CMS) from Jerry Cohen, MD, President, American Society of Anesthesiologists, re CMS-1590-P, RIN 0938-AR11, Medicare Program; Revisions to Payment Policies Under the Physician Fee Schedule, DME Face to Face Encounters, etc. September 4, 2012.
57. American Medical Association Letter to Donald Berwick, Administrator of CMS, RE: Payment Policies Under the Physician Fee Schedule and Other Revisions to Part B for CY 2012; Proposed Rule; 76 Fed. Reg. 42,772 (July 19, 2011); CMS1524-P. August 29, 2011.

58. Wyden R, Ryan P. Guaranteed choices to strengthen Medicare and health security for all: Bipartisan options for the future. http://budget.house.gov/uploadedfiles/ wydenryan.pdf

59. Wilensky GR. Directions for bipartisan Medicare reform. N Engl J Med 2012; 366:1071-1073.

6o. Emanuel EJ, Fuchs VR. Shortening medical training by $30 \%$. JAMA 2012; 307:1143-1144. 